CASE REPORT

\title{
HIV associated Mycobacterium colombiense cervical lymphadenitis: A case report
}

\author{
Paloma Irizarry ${ }^{1}$, Keith Basler ${ }^{2}$, Matthew Mifsud ${ }^{2}$, Cynthia Mayer ${ }^{3}$, Tapan Padhya ${ }^{2}$ \\ ${ }^{1}$ University of South Florida Morsani College of Medicine, Tampa, Florida, USA \\ ${ }^{2}$ Department of Otolaryngology, University of South Florida Morsani College of Medicine; Tampa, Florida, USA \\ Infectious Disease Associates of Tampa Bay, Tampa, Florida, USA
}

\begin{abstract}
We report a case of Mycobacterium colombiense in a human immunodeficiency virus (HIV) positive patient who presented with tender cervical lymphadenopathy that progressed to a fistulous tract. The identification, treatment, and management of infection with this pathogen are discussed. This represents the first case to our knowledge of infection with this organism in the United States. J Microbiol Infect Dis 2017; 7(2): 101-103
\end{abstract}

Keywords: Colombiense, nontuberculous mycobacteria, HIV, lymphadenitis

\section{INTRODUCTION}

The Mycobacterium avium complex (MAC) is a family of common opportunistic nontuberculous mycobacterial (NTM) species that is particularly associated with acquired immunodeficiency disease (AIDS). Mycobacterium avium complex includes pathogen such as Mycobacterium intracellulare, Mycobacterium avium, and $M$. colombiense [1]. $M$. colombiense is a rarely reported member of this family and is referred to as MAC-X. It has been differentiated from other species in this family by the 16S-23S rRNA internal transcribed spacer (ITS-1) sequence. It is most closely related to $M$. avium when compared via gene sequencing; however, it differs from it with its ability to produce a urease [2]. It has been reported to cause lymphadenitis in both immunocompromised and immunocompetent hosts [3-5]. The diagnosis of M. colombiense can be challenging. Speciation can occur due to positive reactivity with commonly used MAC-specific molecular testing, Accuprobe $\AA$ (Gen-Probe) [2,6]. While varied cases have been identified within South America and Western Europe, the true global prevalence is uncertain [6]. We present to our knowledge the first confirmed case of $M$. colombiense, manifesting as cervical lymphadenitis, within the United States.

\section{CASE REPORT}

A 36-year-old male with known history of HIV/AIDS presented originally to the emergency department with a perirectal abscess and fever. Upon examination he was noted to have a large right submandibular lymph node. Laboratory data revealed a baseline CD4 count of $54 / 4 \%$ and a positive viral load at 5 logs. Computed tomography (CT) revealed right level II-V adenopathy, with the largest node measuring 4 . (Figure 1). One month prior to presentation he was started on antiretroviral therapy (ART) with Truvada, Prezista, and Norvir due to progressive HIV infection and low CD4 count. After initiation of ART his CD4 count increased to $193 / 9 \%$.

Fine needle aspiration biopsy was performed and was analyzed via PCR, revealing Mycobacterium avium intracellulare lymphadenitis. Antimycobacterial therapy was initiated with levofloxacin, ethambutol, and azithromycin. Despite this approach, cervical adenopathy continued to worsen with the patient ultimately developing a sinus tract to the overlying neck skin. The aspirate was then sent to the National Jewish Health Laboratory for repeat gene sequencing to obtain more detailed infectious disease and sensitivity information. The pathogen was re-characterized as a

Correspondence: Paloma Irizarry B.A. University of South Florida Morsani College of Medicine, Tampa, Florida, USA 
multidrug resistant strain of $M$. colombiense. In review of antibiotic sensitivities by minimum inhibitory concentration (MIC) the organism was found to be fully sensitive to clarithromycin and clofazimine; intermediately sensitive to amikacin, ethambutol and rifampin; and resistant to ciprofloxacin and rifabutin (Table 1).

Table 1: Antibiotic sensitivities for M. colombiense.

\begin{tabular}{|l|c|}
\hline Antibiotic & $\begin{array}{c}\text { Minimum inhibitory } \\
\text { concentration (mcg/ml) }\end{array}$ \\
\hline $\begin{array}{l}\text { Ciprofloxacin } \\
\text { (resistant) }\end{array}$ & $>4.0(\mathrm{mcg} / \mathrm{ml})$ \\
\hline $\begin{array}{l}\text { Clofazimine } \\
\text { (susceptible) }\end{array}$ & 0.12 \\
\hline Rifabutin (resistant) & $>0.5$ \\
\hline $\begin{array}{l}\text { Rifampin } \\
\text { (intermediate) }\end{array}$ & 4.0 \\
\hline $\begin{array}{l}\text { Ethambutol } \\
\text { (intermediate) }\end{array}$ & 4.0 \\
\hline $\begin{array}{l}\text { Streptomycin } \\
\text { (intermediate) }\end{array}$ & 4.0 \\
\hline $\begin{array}{l}\text { Amikacin } \\
\text { (intermediate) }\end{array}$ & 4.0 \\
\hline $\begin{array}{l}\text { Clarithromycin } \\
\text { (susceptible) }\end{array}$ & $\leq 4.0$ \\
\hline
\end{tabular}

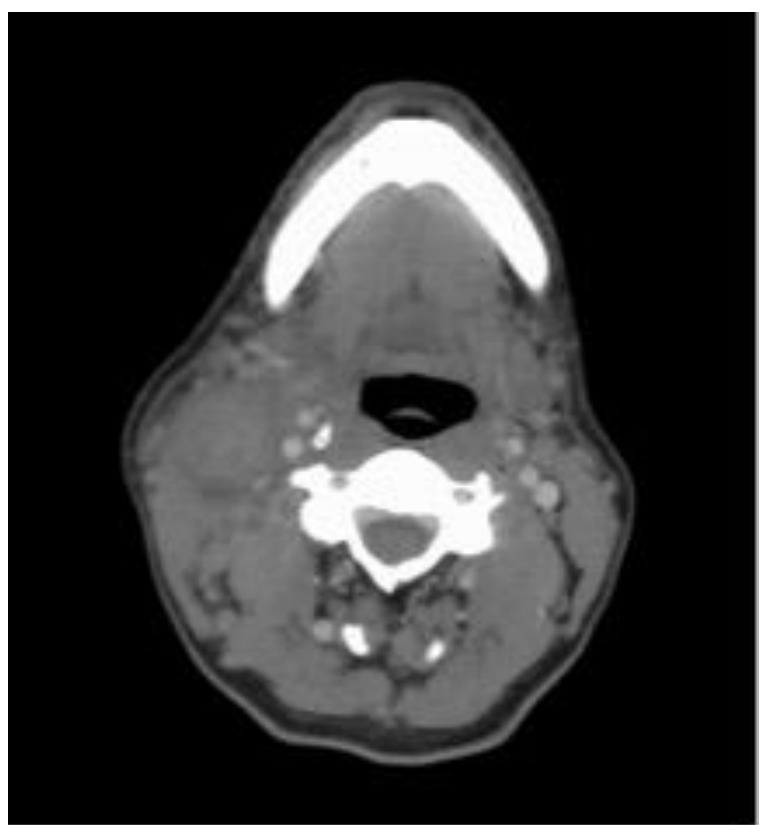

Figure 1. Axial Computed Tomography showing post operative enlarged right lymph nodes

A course of at least nine months of clarithromycin $500 \mathrm{mg}$ twice a day, ethambutol $1200 \mathrm{mg}$ once daily, and clofazimine $100 \mathrm{mg}$ once daily was thus initiated along with amikacin $500 \mathrm{mg}$ daily for six weeks. Despite radiographic resolution of his adenopathy, he continued to have a persistent right level IV sinus tract requiring surgical excision (Figure 2). Surgical resection of the sinus tract, surrounding neck and residual fibrotic cervical adenopathy was thus undertaken for clearance of residual disease. In continuing following the site has healed well without evidence of persistent/recurrent mycobacterial infection. The patient was unfortunately lost to follow up after nine months of treatment.

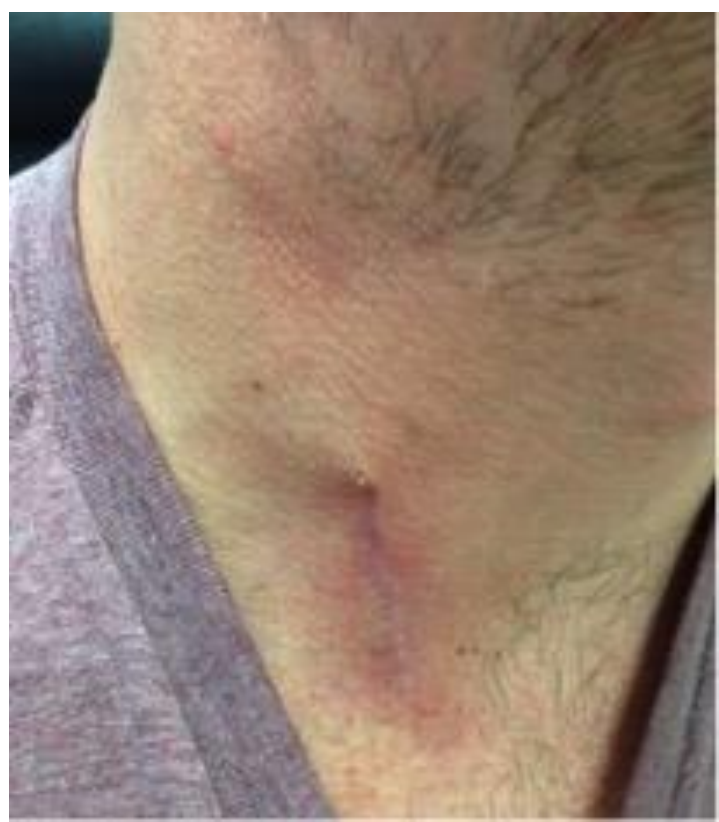

Figure 2. Photograph showing post operative external opening of right level IV sinus tract

\section{DISCUSSION}

Rates of opportunistic infections in patients with HIV have decreased with the use of highly active antiretroviral therapy (HAART) [4]. Although HAART has led to reduced rates of these infections in HIV positive patients, NTM remains a common cause of infection in patients with AIDS [4]. This patient most likely had developed opportunistic infections due to his very poor immune system being reconstituted. He likely became symptomatic with the mycobacterial infection when his CD4 count rose, indicating an immune reconstitution inflammatory response syndrome (IRIS).

The incidence of disseminated MAC infections in HIV patients on adequate ART with CD4 count less than $100 / \mathrm{mm} 3$ is 2 per 100 -persons annually [7]. Contrastingly, in patients not on ART with AIDS-associated immunosuppression, the incidence of MAC infection rises to $20-40 \%$ 
(6). Infection is typically acquired via inhalation, ingestion, or direct pathogen invasion [3]. Common disseminated symptoms include persistent fevers, night sweats, and weight loss. Localized disease, however, can present as cervical or mesenteric lymphadenitis, osteomyelitis, and skin or soft tissue abscesses [7]. The diagnosis of disseminated MAC is achieved utilizing specific molecular probes, or mycobacterial blood cultures (in the setting of disease dissemination). A multi-drug antibiotic regimen, commonly clarithromycin with ethambutol, is commonly utilized as a first line treatment $[1,7]$.

Accurate identification of different MAC species, for example $M$. colombiense, is important in order to ensure effective disease treatment. The gold standard for diagnosis involves nucleotide sequencing of the 16S rRNA gene [6]. Alternatively, nucleotide sequencing of rpoB genes, or internal transcribed spacer with the urease test may also be used [6]. Treatment options are both medical and surgical. Medical therapy includes both antibiotics as well as antiretroviral therapy for those with immunodeficiency [5]. Antibiotic selection is tailored to sensitivity analysis; multi-drug resistance is a particular concern as reflected in our case. Due to rising antibiotic resistance, multiple antibiotics are used concomitantly for a minimum of 3-6 months $[5,8]$. Typical regimens might include combinations of the following: clarithromycin or azithromycin as initial antibiotic; ethambutol as the second additional antibiotic; and clofazimine, rifampin, rifabutin, ciprofloxacin, or amikacin as the third antibiotic if necessary [5]. Surgical excision will often be necessary for "localized" sources of infection as these tend to respond poorly to simple incision/drainage procedures and will often fail to respond to antibiotic therapy alone [1].

\section{Conclusion}

This case represents a rare occurrence of NTM lymphadenitis by a novel pathogen in a HIV positive adult male. To the best of our knowledge, this is the first well-reported case of M. colombiense, occurring within the United States. We demonstrate both the difficulty and importance of accurately speciating mycobacterial organisms to ensure effective disease treatment. We also affirm the importance of a multi-disciplinary approach (both medical and surgical therapy) to ensure complete eradication of disease within the infected cervical lymph node basins.

\section{ACKNOWLEDGEMENTS}

Declaration of conflicting interests: No competing interests to declare.

Funding: The authors received no financial support for the research and/or authorship of this article.

\section{REFERENCES}

1. Flint D, Mahadevan M, Barber C, et al. Cervical lymphadenitis due to non-tuberculous mycobacteria: Surgical treatment and review. Int $\mathrm{J}$ Pediatric Otorhinolaryngology 2000; 53:187-194

2. Murcia, MI, Tortoli E, Menendez, MC, Palenque E, Garcia MJ. Mycobacterium colomiense sp. nov., a Novel Member of the Mycobacterium avium complex and Description of MAC-X as a New ITS Genetic Variant. Int J Syst Evol Microbiology 2006; 56:2049-2054.

3. Esparcia Ó, Navarro F, Quer M, Coll P. Lymphadenopathy Caused by Mycobacterium colombiense. J Clin Microbiology 2008; 46 (5):1885-1887.

4. Jones D, Havlir DV. Nontuberculous Mycobacteria in the HIV Infected Patient. Clin Chest Med 2002; 23:665-674.

5. Starke JR, Correa AG. Management of Mycobacterial Infection and Disease in Children. Pediatr Infect Dis J 1995; 14 (6):455-469.

6. Leguizamón J, Hernández J, Murcia MI, Soto CY. Identification of Potential Biomarkers to Distinguish Mycobacterium colombiense from other Mycobacterial species. Mol Cell Probes 2013; 27 (1):46-52.

7. Center for Disease Control and Prevention. (2009). Guidelines for Prevention and Treatment of Opportunistic Infections in HIV-Infected Adults and Adolescents. Retrieved from http://www.cdc.gov/mmwr/preview/mmwrhtml/rr58 e324a1.htm. Accessed 02/25/2016

8. Ramos-e-Silva M, Ribeiro de Castro MC. Mycobacterial Infections. In: Bolognia JL, Jorizzo JL, Rapini RP, editors. Dermatology, $2^{\text {nd }}$ edn. London: Elsevier Ltd 2008; 1119-1120. 